\title{
BMJ Open Effects of tai chi on postural control during dual-task stair negotiation in knee osteoarthritis: a randomised controlled trial protocol
}

\author{
Xiangbin Wang, ${ }^{1,2}$ Meijin Hou (D) , ${ }^{2,3}$ Shaoqing Chen, ${ }^{1,2}$ Jiao Yu, ${ }^{1,2}$ Dalu Qi, ${ }^{4}$ \\ Yanxin Zhang, ${ }^{5}$ Bo Chen, ${ }^{1,2}$ Feng Xiong, ${ }^{1,2}$ Shengxing Fu (i) , ${ }^{1,2}$ Zhenhui Li, ${ }^{1,2}$ \\ Fengjiao Yang, ${ }^{1,2}$ Alison Chang, ${ }^{6}$ Anmin Liu, ${ }^{7}$ Xuerong $\mathrm{Xie}^{8}$
}

To cite: Wang X, Hou M, Chen S, et al. Effects of tai chi on postural control during dual-task stair negotiation in knee osteoarthritis: a randomised controlled trial protocol. BMJ Open 2020;10:e033230. doi:10.1136/ bmjopen-2019-033230

- Prepublication history for this paper is available online. To view these files, please visit the journal online (http://dx.doi. org/10.1136/bmjopen-2019033230).

$\mathrm{XW}$ and $\mathrm{MH}$ are joint first authors.

Received 13 August 2019 Revised 30 November 2019 Accepted 03 December 2019

Check for updates

(C) Author(s) (or their employer(s)) 2020. Re-use permitted under CC BY-NC. No commercial re-use. See rights and permissions. Published by BMJ.

For numbered affiliations see end of article.

Correspondence to

Dr Xuerong Xie;

384098067@qq.com

\section{ABSTRACT}

Introduction Stair ascent and descent require complex integration between sensory and motor systems; individuals with knee osteoarthritis (KOA) have an elevated risk for falls and fall injuries, which may be in part due to poor dynamic postural control during locomotion. Tai chi exercise has been shown to reduce fall risks in the ageing population and is recommended as one of the nonpharmocological therapies for people with KOA. However, neuromuscular mechanisms underlying the benefits of tai chi for persons with KOA are not clearly understood. Postural control deficits in performing a primary motor task may be more pronounced when required to simultaneously attend to a cognitive task. This single-blind, parallel design randomised controlled trial (RCT) aims to evaluate the effects of a 12-week tai chi programme versus balance and postural control training on neuromechanical characteristics during dual-task stair negotiation. Methods and analysis Sixty-six participants with KOA will be randomised into either tai chi or balance and postural control training, each at 60 min per session, twice weekly for 12 weeks. Assessed at baseline and 12 weeks (ie, postintervention), the primary outcomes are attention cost and dynamic postural stability during dual-task stair negotiation. Secondary outcomes include balance and proprioception, foot clearances, self-reported symptoms and function. A telephone follow-up to assess symptoms and function will be conducted at 20 weeks. The findings will help determine whether tai chi is beneficial on dynamic stability and in reducing fall risks in older adults with $\mathrm{KOA}$ patients in community.

Ethics and dissemination Ethics approval was obtained from the Ethics Committee of the Affiliated Rehabilitation Hospital of Fujian University of Traditional Chinese Medicine (\#2018KY-006-1). Study findings will be disseminated through presentations at scientific conferences or publications in peer-reviewed journals. Trial registration number ChiCTR1800018028.

\section{INTRODUCTION}

The prevalence of knee osteoarthritis (KOA) has risen significantly over the past 30 years in China. ${ }^{1}$ A 2012 national survey conducted by the US Centers for Disease Control and

\section{Strengths and limitations of this study}

- Aiming to examine the potential neuromuscular mechanisms underlying the benefits of tai chi for balance and postural control, this study will assess both biomechanical and self-reported outcomes, rather than self-reported outcomes alone.

- An active control group receiving balance and postural control training, following the same dosage as the tai chi group, enables comparisons between two intervention strategies.

- Employing the dual task paradigm, which simulates everyday dual demands of motor and cognitive interaction and adds complexity to a single motor task, may accentuate tai chi-induced changes in balance and postural control.

- As a single-blind randomised controlled trial, in which participants are aware of group assignment, participant expectation may introduce bias.

- This study is to compare the effects of tai chi and balance and posture training on dynamic stability for knee osteoarthritis, thus there is no true control group setting.

Prevention showed, among people aged 45 years and older, individuals with arthritis had a 2.4-fold increased risk of $\geq 2$ falls and a 2.5fold increased risk of fall injuries in the past year, compared with those without arthritis. ${ }^{2}$ Stair ascent and descent need more complex integration between sensory and motor systems, with increased potential for trips, slips and subsequent falls, resulting in more severe injuries than falls on level ground. ${ }^{3}$ The mechanisms and contributors underlying the occurrence of falls in individuals with $\mathrm{KOA}$ are not well understood and most likely multfactorial. Both concentric and eccentric contractions of lower limb muscles are required during descent or ascend to different extents. For example, quadriceps contract concentrically and hamstring 
contract eccentrically when foot strike on ascending but more eccentric contraction on quadriceps and concentric contraction on hamstring on supporting leg before the other foot touch the down step during descending. During stair climbing or descent, people with KOA both have altered kinematics changes in sagittal plane and delayed quadriceps muscle activity. ${ }^{45}$ Impaired postural control and balance, reduced knee muscle strength, poor proprioceptive acuity and joint symptoms have been postulated to elevate fall risks in this population. ${ }^{67}$ Therapeutic strategies addressing these potential underlying neuromechanical factors have been advocated to prevent falls. $^{78}$

Tai chi, a traditional Chinese fitness exercise aiming at stimulating and integrating musculoskeletal, sensory and cognitive systems through a series of stabilising and destabilising movements and postural actions, can simultaneously improve postural control, balance, proprioception and lower limb muscle strength in older adults. ${ }^{9-11}$ It is one of the non-pharmacological therapies recommended by the American College of Rheumatology for the treatment of KOA. ${ }^{12}$ Tai chi has also been shown to reduce fall risk in the ageing population. ${ }^{13}$ Despite common recommendations of tai chi for balance control and fall reduction, the neuromechanical mechanisms underlying these benefits are not clearly understood.

Postural control and stability require sensorimotor processing and cognition. ${ }^{14} 15$ The extra consumption of the cognitive resource negatively influences postural maintenance during locomotion, increasing stride time variability and decrease in minimum foot clearance were found during stair walking, ${ }^{16}$ may lead to greater safety concerns while negotiating stairs. In other words, there is a cognitive attention cost while performing a dual task of a primary motor task plus a secondary cognitive task, such as talking or texting during stair negotiation. The dualtask attention cost is commonly computed as the difference in task performance (eg, time, speed or accuracy) between single primary task vs dual task. ${ }^{18}$ In persons with severe KOA, achieving greater knee pain reduction after arthroplasty was associated with decrease in dualtask attention cost of gait variability during walking on a long track. ${ }^{19}$ Compared with asymptomatic controls, those with KOA exhibited poor dynamic postural control and recovery during a simulated forward fall; these deficits were more pronounced when required to simultaneously attend to a cognitive task. ${ }^{20}$ The dual task paradigm is effective in revealing intervention-related changes in postural control than the primary motor task alone.

To date, few studies have assessed the effects of tai chi on balance and postural control using motor cognitive dual tasks in persons with KOA. Our hypothesis is tai chi practice would have no less effect than balance training, which could be presented by dual task performance, and associated with improved balance ability. The planned single-blind, parallel design randomised controlled trial (RCT) aims to evaluate the effects of a 12-week tai chi programme versus balance and postural control training (control group) on attention cost and dynamic postural stability during stair negotiation. Secondary outcomes include balance control and proprioceptive acuity, foot clearance and patient-reported symptoms and function. Findings of the proposed project will elucidate potential neuromuscular mechanisms underpinning the benefits of tai chi.

\section{METHODS AND ANALYSIS}

\section{Trial design}

This study is a single-blind, parallel design RCT conducted at the Affiliated Rehabilitation Hospital of Fujian University of Traditional Chinese Medicine (FJTCM) in China. The study protocol follows the Standard Protocol Items: Recommendations for Interventional Trials. ${ }^{21}$

\section{Participants}

Sixty-six participants with KOA will be recruited from the community or the outpatient department of the Affiliated Rehabilitation Hospital of FJTCM. Participants will be recruited via posters on community bulletin boards, mailed leaflets and advisements at the hospital recruiting station. After initial screening for inclusion and exclusion criteria, eligible individuals will receive oral and written information about this trial from two trained research assistants. Those who agree to participate will sign a written informed consent and be scheduled for the preintervention baseline assessment.

Inclusion criteria ${ }^{2223}$ :

1. Adults aged 38 and 80 years.

2. Confirmed clinical diagnosis of unilateral or bilateral KOA in accordance with the 2010 Clinical Diagnostic Criteria for the Diagnosis and Treatment of Osteoarthritis by the Chinese Medical Association Rheumatology Branch. Specifically, the diagnosis is confirmed by meeting items $1+2+3+4$, items $1+2+5$ or items $1+4+5$ (item 1: knee pain most of the time in the past month; item 2: crepitus; item 3: morning stiffness lasting $\leq 30 \mathrm{~min}$; item 4 : age $\geq 38$ years; and item 5 : palpable bony enlargement).

3. Report average knee pain over the past week $\geq 25$ on a $100 \mathrm{~mm}$ visual analogue scale.

4. Definite unilateral or bilateral tibiofemoral osteophytes by radiograph (ie, at least Kellgren/Lawrence (K/L) grade 2).

5. Able to walk on level ground for a minimum of 6 and ascend/descend eight steps of stairs without assistive devices.

6. No history of musculoskeletal pathology or trauma in the past 6 months.

Exclusion criteria ${ }^{20} 24$.

1. Advanced radiographic disease $(\mathrm{K} / \mathrm{L}$ grade 4$)$ in either knee.

2. Acute, infectious diseases or other systemic diseases.

3. Gait patterns affected by another disease of the nervous or musculoskeletal system, such as stroke, fractures and rheumatoid arthritis. 


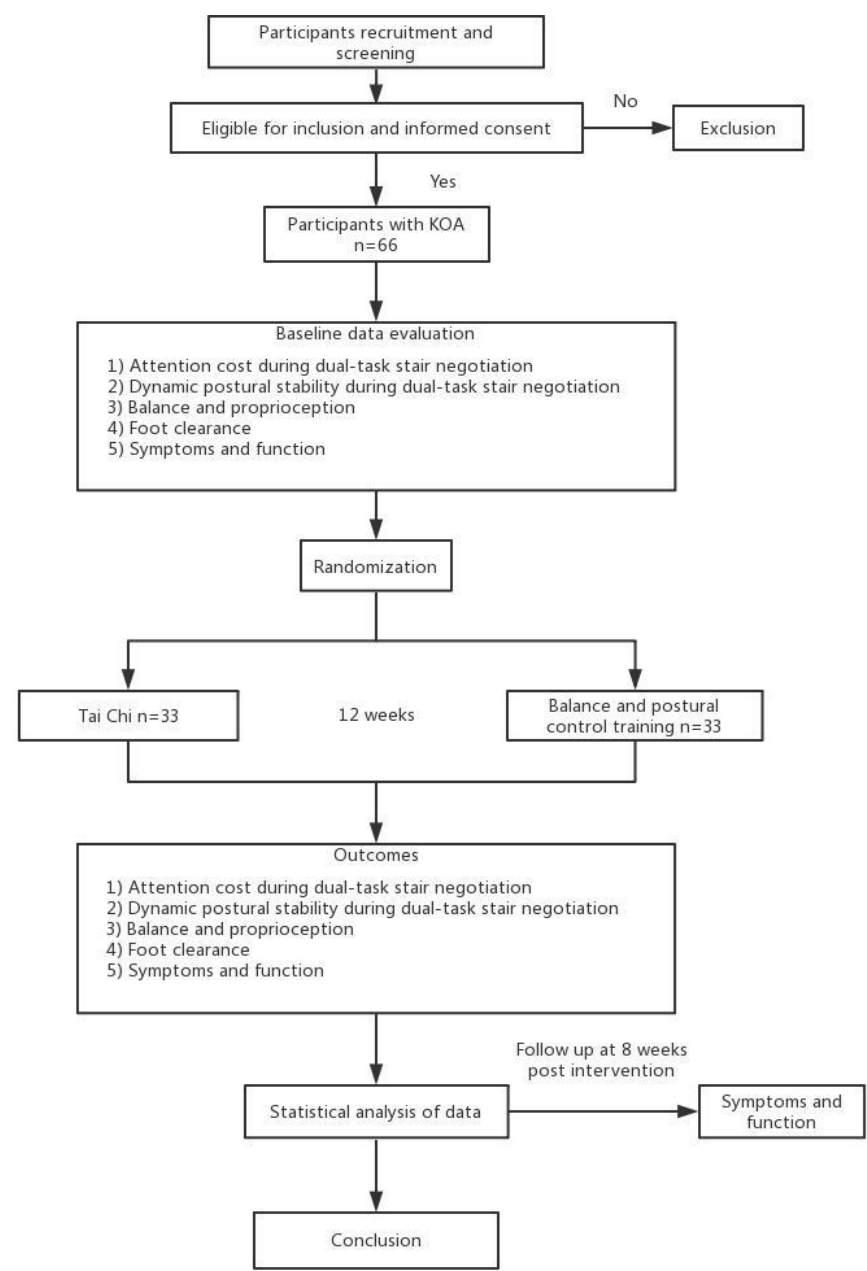

Figure 1 CONSORT flow chart of study design. CONSORT, Consolidated Standards of Reporting Trials; KOA, knee osteoarthritis.

4. A planned intra-articular steroid injection or arthroscopic surgery in the next 3 months, intra-articular hyaluronic acid injection in 6 months and total knee replacement in 12 months.

5. Recent treatments by modern or traditional Chinese medicine rehabilitation methods in the past 2 weeks.

6. Severe cognitive impairment (Montreal Cognitive Assessment $(<18)$ ).

7. Allergic to medical alcohol and adhesive.

8. Other reasons for exclusion (eg, unable to participate due to lack of transportation or schedule conflict).

\section{Procedure}

A Consolidated Standards of Reporting Trials (CONSORT) flow chart of the study design is shown in figure 1. Sixty-six participants with KOA would be randomised into tai chi (intervention) or balance and postural control training (control) group. The intervention group will participate in a $60 \mathrm{~min}$ tai chi exercise programme, twice a week for 12 weeks, while the control group will execute balance training following the same treatment duration and frequency as the tai chi group. The primary outcomes are attention cost and dynamic postural stability during dual-task stair negotiation; secondary outcomes are balance and proprioception, foot clearances, self-reported symptoms and function. A telephone follow-up to assess symptoms and function by self-report will be conducted 8 weeks after conclusion of the intervention.

\section{Randomisation and allocation concealment}

Participants meeting the inclusion/exclusion criteria and giving informed consent will be randomised to one of the two groups after preintervention baseline assessment. Random sequences of 1-66 will be generated by the IBM SPSS Statistics V.20. Through a random number generator, the random seed is set up at the default value of 20000000 . Random numbers are generated and then arranged in ascending order; 1-33 will be classified as the tai chi group and 34-66 as the control group. An independent research assistant who is not involved in recruitment, evaluation and intervention will inform eligible participants of their treatment assignment via telephone prior to intervention commencement.

\section{Blinding}

The nature of the study intervention does not permit blinding participants with regard to group assignment. To minimise bias, participants will be told that the exercise intervention they are assigned to will improve physical function and quality of life. The outcome assessors, data managers and statistical analysts will be blinded to group assignment; the numbers 1 and 2 will be used to code the respective treatment group. After the statistical analyses are completed, the grouping code will be revealed by the project manager.

\section{Interventions}

Tai chi group

Ten-form tai chi programme is easy to learn and suitable for group practice, it consists of 10 movements: (1) commencement, (2) repulse monkey, (3) brush knee twist step, (4) parting the wild horse's mane, (5) wave hands like clouds, (6) golden cock stands one leg, (7) kick with heel leading, (8) grasp the peacock's tail, (9) cross hand and (10) closing (see figure 2). ${ }^{25}$ Tai chi is a series of individual movements linked together to flow smoothly from one form to another. When performed with continuity, these postures and movements effectively involve all body regions and engage controls of postural alignment, balance and coordination. Those in the tai chi group will participate in a 12-week tai chi exercise training, 60 min per session, twice a week. The hour-long session includes $10 \mathrm{~min}$ warm-up, $40 \mathrm{~min}$ exercise and $10 \mathrm{~min}$ cool-down. To implement a standardised Yang's 10-form tai chi programme, a qualified tai chi instructor from the FJTCM with 10 years of teaching experience will lead the group with a maximum of 12 participants per group at the Ping Shan campus of FJTCM. 


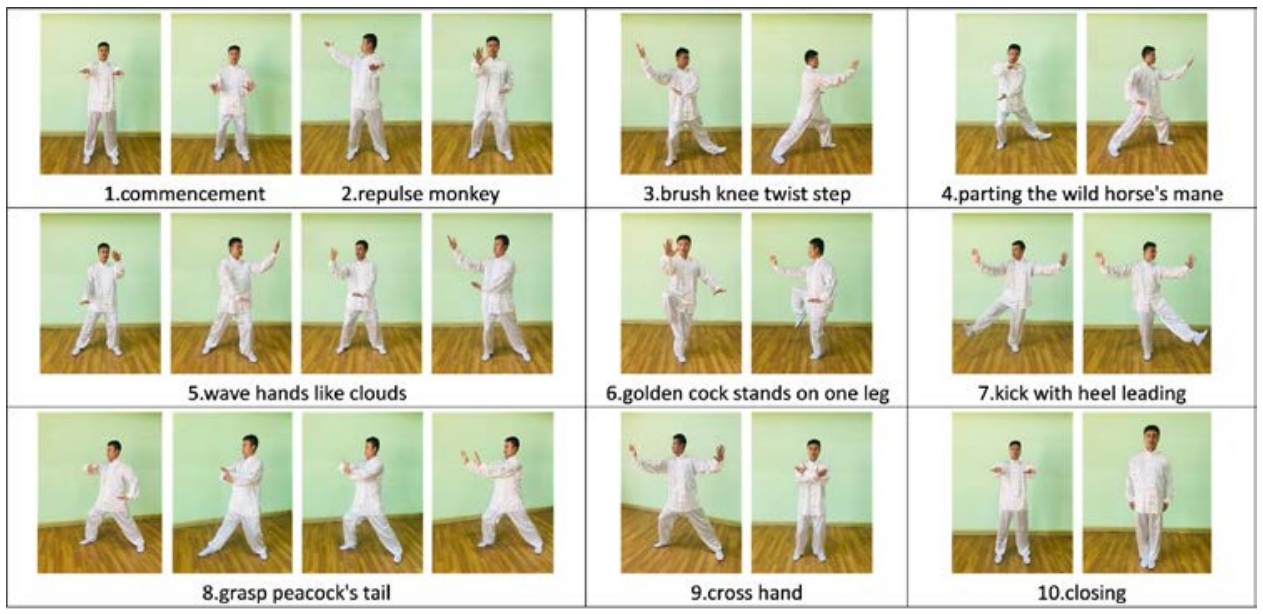

Figure 2 Ten-Form tai chi.

\section{Balance and postural control training group}

Participants in the balance and posture control training group will be trained in a group by experienced rehabilitation physicians or physical therapists, twice weekly over 12 weeks, ${ }^{26}$ at the Affiliated Rehabilitation Hospital of FJTCM. The training session includes warm-up for about $10 \mathrm{~min}$, balance exercises for $40 \mathrm{~min}$ and cooldown for $10 \mathrm{~min}$. The balance exercises will include five parts: (1) single leg standing; (2) walking forward; (3) walking backward; (4) side stepping; and (5) single leg tapping. ${ }^{27}$ Parts $1-4$ would be exercised in every session, part $(5$ will be added when participants are capable to work. Progress in each part of balance exercises would follow the principles like increasing duration increments, changing supporting plane from firm surface to soft foam, decreasing visual input (from eyes open to close) or decreasing area of support base (foot to toes). ${ }^{27}$ After weekly assessment, if the participants could complete the previous level, they could be trained to upgrade into the next level. If they feel too pain (eg, VAS $>4$ ) or too difficult to work in the advanced level, the training would be maintained in the current level or even downgraded to previous level, or take longer break during intervals.

After completing the above two 12-week programmes, all participants will be advised to continue tai chi or balance and postural control training at home for 1 hour at least twice a week, punch the clock by uploading exercise videos each time in the following 8 weeks.

\section{Outcome measurement}

Primary outcome: attention cost during dual-task stair negotiation Each participant should complete a single stair task (SST), a single cognitive task and a dual stair task (DST). The order of different tasks will be randomised.

\section{Single stair task}

Barefoot participants will be instructed to climb and walk down a eight-step customised staircase five times at a self-selected comfortable pace, two feet cannot fall on the same step at the same time. The handrailed staircase features $604.5 \mathrm{~mm}$ wide steps, each with a $177.8 \mathrm{~mm}$ rise and $264.1 \mathrm{~mm}$ run; there is enough landing space (100 $\mathrm{mm} \times 100 \mathrm{~mm}$ ) to allow the participant to turn around. A wireless timing system (Brower Timing Systems, LLC, USA) will be used to record the time required to complete the eight-step stair ascent and eight-step stair descent, respectively. The stair ascent time will be averaged over five trials; the stair descent time also averaged over five trials.

\section{Single cognitive task}

Participants will perform a counting backward test, subtracting $3 \mathrm{~s}$ from any random number between 20 and 99 generated by the the IBM SPSS Statistics V.20. software while sitting within a fixed time, previously calculated for each participant during stair ascent or descent. ${ }^{28}$

\section{Dual stair task}

Participants will be asked to execute the counting test while stair negotiation. Similarly, the time required to complete the stair ascent and descent will be separately recorded by the wireless timing system.

Based on stair time (ST), the stair performance is evaluated by coefficient of variation of $\mathrm{ST}(\mathrm{CoV})$. $\mathrm{CoV}$ is calculated as the percentage of the quotient between SD and ST mean $(\mathrm{CoV}=[\mathrm{SD} / \mathrm{ST}$ mean $] \times 100)$. Correct calculations (ie, number of correct response) is recorded to analysis cognitive performance. ${ }^{29}$ To compare the motor and cognitive function between single task and dual task, dual-task attention cost is measured as:

I (Single-task-Dual-task) /Single-task $\mid \times 100$

Larger the value, the more attentional resources are required on motor or cognitive performance during dual-task stair negotiation. ${ }^{1830}$

Primary outcome: dynamic postural stability during stair negotiation

During the respective SST and DST, three-dimensional movement kinematics will be collected by Qualisys Motion Capture Systems (10 cameras, Oqus 700+, sampling frequency $100 \mathrm{~Hz}$, Qualisys Trace Manager, Sweden). An experienced lab personnel will place 55 retroreflective 
Table 1 Fifty-five retroreflective markers placement for collecting whole body kinematic data

\begin{tabular}{|c|c|}
\hline Marker name & Marker location \\
\hline & Upper body \\
\hline L/R_HEAD & Just above the ear. \\
\hline SGL & Glabulla. \\
\hline CV7 & Seventh cervical vertebrae. \\
\hline L/R_SIA & Scapula-inferior angle. \\
\hline TV10 & 10th thoracic vertebrae. \\
\hline L/R_SAE & Scapula-acromial edge. \\
\hline L/R_HUM & Lateral surface of the upper arm. \\
\hline L/R_HLE & Humerus - lateral epicondyle. \\
\hline L/R_RSP & Radius - styloid process. \\
\hline L/R_USP & Ulna - styloid process. \\
\hline \multirow[t]{2}{*}{ L/R_HM2 } & Basis of forefinger. \\
\hline & Lower body \\
\hline L/R_IAS & Anterior superior iliac spine. \\
\hline L/R_IPS & Posterior superior iliac spine. \\
\hline L/R_TH1-4 Cluster & $\begin{array}{l}\text { Cluster of four markers placed on the } \\
\text { lateral surface of the thigh. }\end{array}$ \\
\hline L/R_FLE & Lateral epicondyle. \\
\hline L/R_FME & Medial epicondyle. \\
\hline L/R_SK1-4 Cluster & $\begin{array}{l}\text { Cluster of four markers placed on the } \\
\text { lateral surface of the shank. }\end{array}$ \\
\hline L/R_FAL & $\begin{array}{l}\text { Lateral prominence of the lateral } \\
\text { malleolus. }\end{array}$ \\
\hline L/R_TAM & $\begin{array}{l}\text { Medial prominence of the medial } \\
\text { malleolus. }\end{array}$ \\
\hline L/R_FCC & $\begin{array}{l}\text { Aspect of the Achilles tendon } \\
\text { insertion on the calcaneus. }\end{array}$ \\
\hline L/R_FM1 & $\begin{array}{l}\text { Dorsal margin of the first metatarsal } \\
\text { head. }\end{array}$ \\
\hline L/R_FM2 & $\begin{array}{l}\text { Dorsal aspect of the second } \\
\text { metatarsal head. }\end{array}$ \\
\hline L/R_FM5 & $\begin{array}{l}\text { Dorsal margin of the fifth metatarsal } \\
\text { head. }\end{array}$ \\
\hline
\end{tabular}

Markers are placed bilaterally in the upper and lower extremities. $\mathrm{L} / \mathrm{R}$, left/right.

markers on the whole body of participants (for detailed placement, see table 1). Kinematic data will be processed by the low-pass filter (Butterworth $6 \mathrm{~Hz}$ ). Threedimensional kinetics on each step will be synchronously collected by force plates (sampling frequency $1000 \mathrm{~Hz}$, AMTI 400600, USA) secured under the staircase. Motion data (eg, the centre of pressure (COP) and centre of mass (COM)] during stair negotiation will be analysed by MATLAB 2016a (MathWorks Inc, Natick, Massachusetts, USA) to calculate extrapolated centre of mass (XCOM) and the margin of stability (MOS) ${ }^{31}$ MOS reflects postural stability in the respective anterior-posterior (AP)

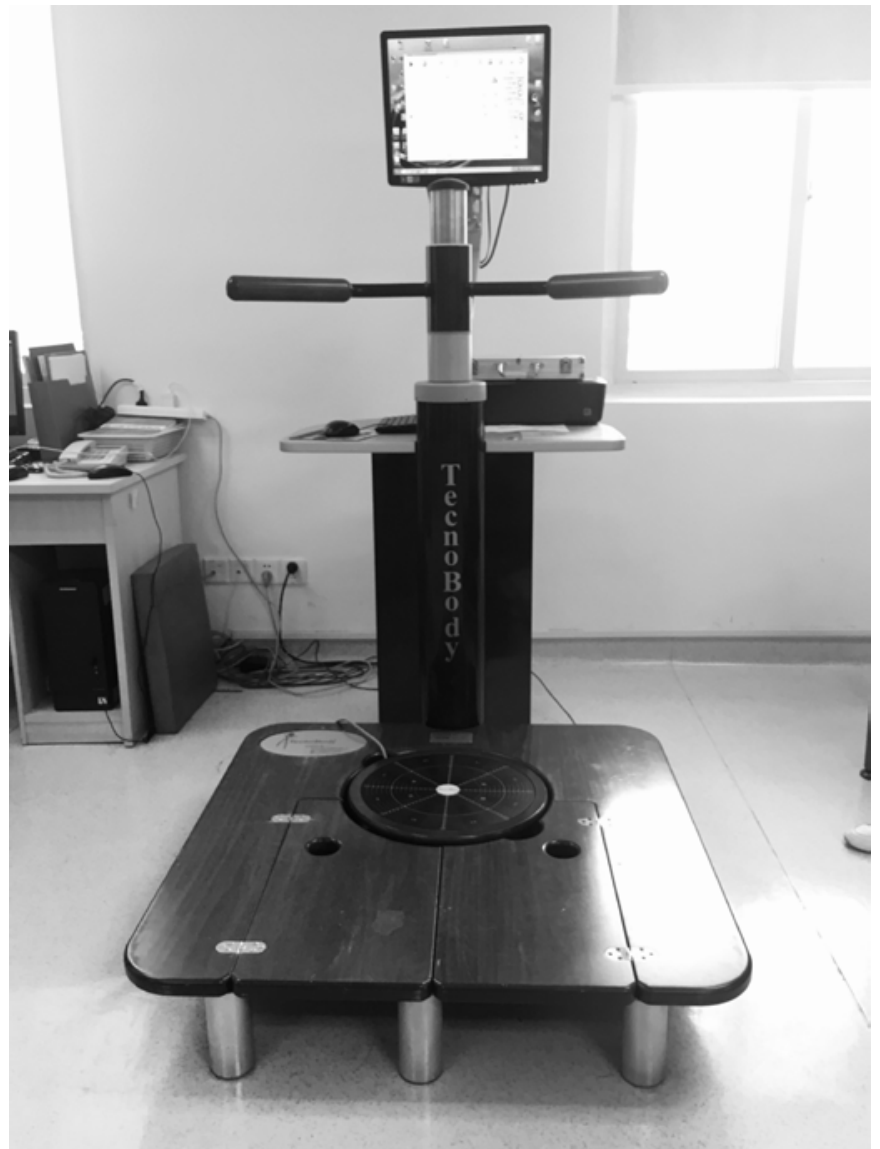

Figure 3 Prokin Balance Trainer (model PK254, TecnoBody, Italy).

and medial-lateral (ML) directions, larger value indicating better stability.

Calculations of XCOM and MOS:

$$
\begin{aligned}
& X C O M=C O M p o s i t i o n+v C O M /\left(g l^{-1}\right)(\text { Equation 1) } \\
& M O S=B O S X C O M \quad(\text { Equation } 2) \\
& \mathrm{g}=9.8 \mathrm{~m} / \mathrm{s}^{2}
\end{aligned}
$$

vCOM: the velocity of COM.

1: the distance between the COP and the COM.

BOS: the border of the base of support.

COMposition: the ML or the AP position of COM, which based on the global coordinate system of the laboratory.

\section{Secondary outcome: balance and proprioception}

Balance control and proprioceptive acuity will be tested by Prokin Balance Trainer (Model PK254, TecnoBody, Italy), which is equipped with a control computer and a $550 \mathrm{~mm} \times 550 \mathrm{~mm}$ static/dynamic sensorised mobile platform (figure 3) and Prokin 3 balance test training software system.

To quantify balance control, the COP during quiet barefoot bipedal standing will be recorded using the Static Stability Module of Prokin System (figure 4). Each participant needs to perform first with eyes open and then with eyes closed, complete three 30 s trials respectively for calculating the average. We will examine the 
length of the COP movement trajectory as well as the area circumscribed by the COP movement trajectory. Greater value indicates worse balance control. ${ }^{32}$

To quantify proprioceptive acuity, the barefoot participant will place the testing foot on an inclined plate in front and the other foot behind on the ground for support, while holding onto supporting posts (figure 5). The participant will be asked to draw five circles within the specified range displayed on a screen in $120 \mathrm{~s}$ by sliding his or her testing foot on the platform. Three trials will be performed for each foot. We will examine the average tracking error (ATE) of each foot. The greater the ATE, the worse the proprioceptive acuity. ${ }^{33}$

\section{Secondary outcome: foot clearance}

Foot clearance is thought to quantify the ability of KOA patients to stair negotiation or through the ground during the swing phase of gait. Minimum toe clearance (MTC) is used to evaluate the risk of the toe contacting the ground when foot clearance, which defines as the vertical lowest event of the toe from toe off to heel on. ${ }^{34}$ A low mean MTC combined with MTC variability potentially causes falling during the swing phase of gait, which is an important event to measure the risk of falls. ${ }^{35}$

\section{Secondary outcome: symptoms and function}

To evaluate the pain, stiffness and function before and after intervention, we will use the Western Ontario and McMaster Universities Arthritis Index (WOMAC), a validated, self-administered, visual analogue scale. ${ }^{36} 37$ The WOMAC scale consists of 24 items, 5 questions for pain, 2 for stiffness and 17 for physical function. Using a $100 \mathrm{~mm}$ visual analogue ruler, of which 'no pain, stiffness, or difficulty' and 'extreme pain, stiffness, or difficulty' are labelled at both ends, each participant will mark on the line according to the extent of the problem for each WOMAC item. Each subscore is determined by the distance (in $\mathrm{mm}$ ) from the origin to the marked point. The pain score range is $0-500,0-200$ for stiffness and $0-1700$ for function. A higher score indicates worse status.

\section{Adherence and safety evaluation}

At first, detailed exercise content and benefits will be informed to the participants; they have the freedom to choose whether to participate. Each instructor is supposed to carefully guide exercise and correct inappropriate movements. We also provide teaching videos to encourage the participants to exercise at home. The number of exercise, leave requests and the corresponding reasons will be documented and reported, as well as any unexpected or adverse events during the study, including time, severity, duration, solutions and outcomes. Participants will be monitored weekly during the 12-week programme and 8-week follow-up.

\section{Sample size}

Stair ascent plus descent time is highly related to the primary outcome of attention cost during stair negotiation. Based on 12.7 $\pm 0.4 \mathrm{~s}^{38}$ (aerobic exercise group) versus

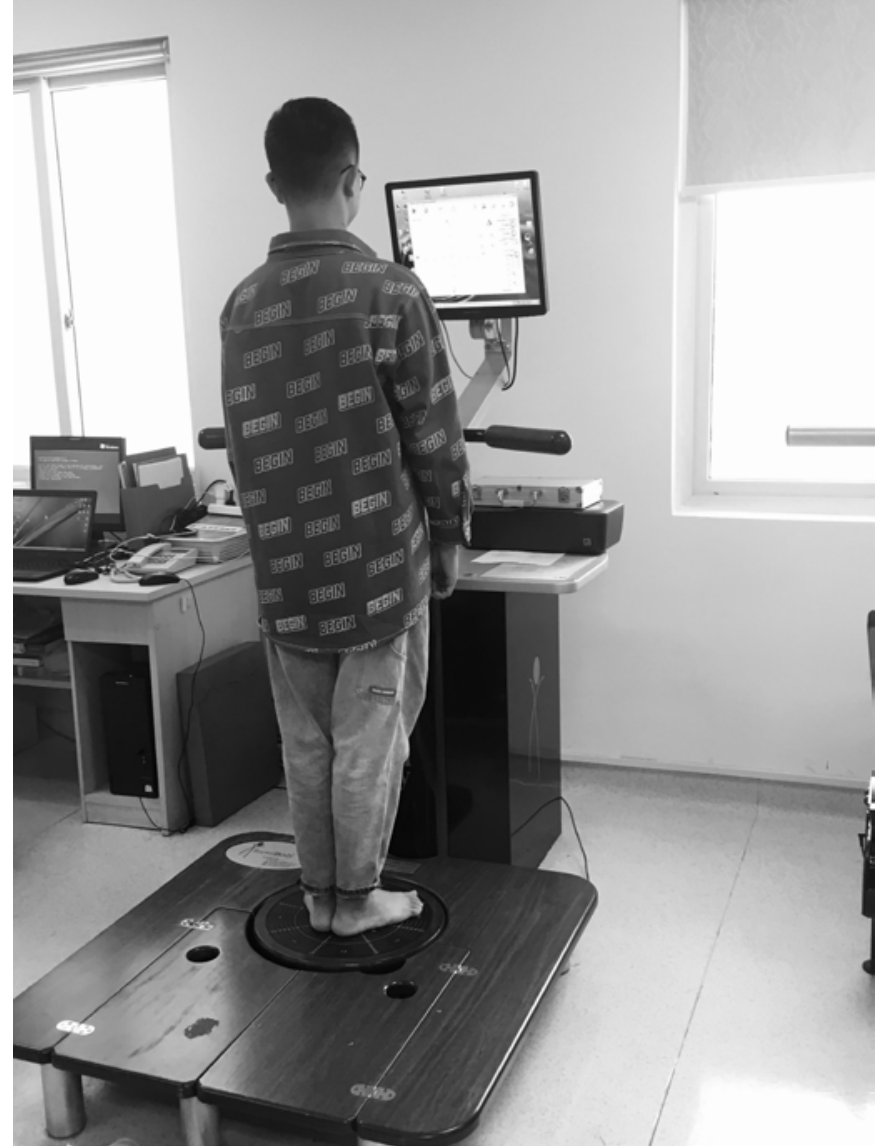

Figure 4 Balance test on Prokin system.

$12.2 \pm 1.8 \mathrm{~s}^{39}$ (control group) for stair ascent plus descent time in participants with KOA, we obtained an effect size of 0.38 (GPower 3.1 software). A sample size of 26 in each of the two groups will have a minimum detectable effect size of 0.4 , with $80 \%$ power and two-sided $\alpha=0.05$ for statistical significance. Assuming 20\% attrition rate, we plan to recruit 66 participants (33 in each group).

\section{Statistical analysis}

IBM SPSS Statistics V.20 will be used for data analyses. Main comparative analyses between groups will be performed using intent-to-treat analysis. If there are some missing data, we will assume that all of them are subjecting to multivariate normal distribution and adopt multiple imputation approaches. Analsysis of covariance(ANCOVA) will be used to compare the differences between two groups with the baseline data as the covariate. Effect size will be calculated for all outcomes with an effect size of 0.2 considered small, 0.5 medium and 0.8 large. The significant level is set as alpha $=0.05$. A value of $p<0.05$ is considered significant.

\section{Data management and monitoring}

WX and HM are principal investigators who will be responsible for participant recruitment, data collection, data management and maintaining confidentiality. All data will be independently entered by a research staff and a duplicate copy will be stored in a separate 


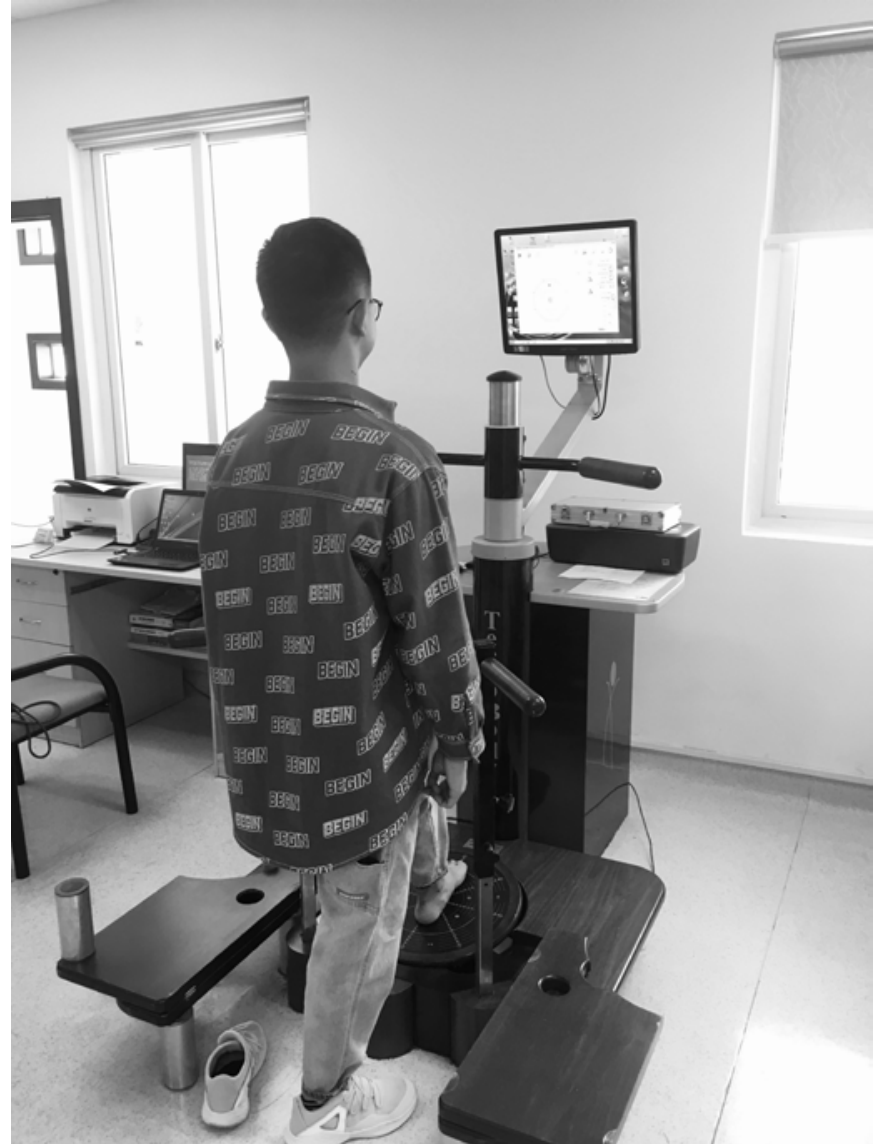

Figure 5 Proprioceptive test on Prokin system.

password-protected hard drive. All computers and electronic systems are kept in locked offices and laboratories and their access restricted only to the research team. Strong passwords consist of letters and number, which are used and changed quarterly and known only to study team members. Any unexpected or adverse events will be reported, documented and reviewed. The data support the findings of this study will be available on request from the corresponding author XX.

\section{Patient and public involvement}

This trial is currently in the recruitment phase. No participant has been involved in the trial. All participants are expected to complete the training by June 2020 .

\section{Ethics and dissemination}

This study protocol is conformed to the principles of the Declaration of Helsinki, which has been approved by the Ethics Committee of the Affiliated Rehabilitation Hospital of FJTCM and registered on the Chinese Clinical Trial Registry website (http://www.chictr.org.cn with the identifier number ChiCTR1800018028). All participants will be informed of the study background as well as potential benefits and risks, and then give informed consent prior to study participation. Study results will be first shared with each participant and disseminated through presentations at scientific conferences and publications in peer-reviewed journals.

\section{DISCUSSION}

The findings will help determine whether tai chi is superior to regarding effects on dynamic stability in people with KOA. The benefits of tai chi programme for KOA have been confirmed, but mostly by self-reported outcomes of symptoms and function. Tai chi's effects on balance and postural control and related neuromechanical characteristics have not been fully explored. We have outlined theoretical premises and presented the study protocols of a single-blind, parallel-designed RCT in persons with KOA. Using the dual-task paradigm during a stair negotiation task, we will evaluate whether a 12-week tai chi programme improves postural control, defined as lower attention cost and better dynamic postural stability postintervention. Additionally, we expect: (1) improved balance control and proprioceptive acuity, assessed by Prokin Balance Trainer; and (2) symptom relief and functional gain, assessed by WOMAC. Study findings will provide important insight into the neuromechanical benefits of tai chi in the setting of KOA and ultimately inform community efforts in reducing fall risks in older adults with chronic knee symptoms. We suppose to promote tai chi in the community in the future if this exercise could improve the dynamic stability and relieve clinical symptoms of KOA patients in the future. At the same time, we will continue our study in patients with early KOA or people at high risk of KOA, exploring the effect of tai chi in delaying or preventing the progression of KOA.

\section{Author affiliations}

${ }^{1}$ College of Rehabilitation Medicine, Fujian University of Traditional Chinese Medicine, Fuzhou, China

${ }^{2}$ Key Laboratory of Orthopedics \& Traumatology of Traditional Chinese Medicine and Rehabilitation (FuJian University of Traditional Chinese Medicine), Ministry of Education, Fuzhou, China

${ }^{3}$ National Joint Engineering Research Center of Rehabilitation Medicine Technology, Fujian University of Traditional Chinese Medicine, Fuzhou, China

${ }^{4}$ College of Sports, Fujian University of Traditional Chinese Medicine, Fuzhou, China ${ }^{5}$ Department of Sport and Exercise Science, The University of Auckland, Auckland, New Zealand

${ }^{6}$ Department of Physical Therapy and Human Movement Sciences, Northwestern University Feinberg School of Medicine, Chicago, Illinois, USA

${ }^{7}$ School of Health and Society, University of Salford, Salford, UK

${ }^{8}$ Rehabilitation Department of the Affiliated 3rd Peoples' Hospital, Fujian University of Traditional Chinese Medicine, Fuzhou, China

Contributors XW conceived, designed and obtained funding for the study. $\mathrm{MH}$ and JY drafted the manuscript and will lead, under the guidance of XW, implementation of the study. SC, XX, DQ, YZ, BC, FX, SF, ZL, FY, AC and AL contributed to the study protocol, including intervention programme design, outcome measures collection and data analysis. All authors were involved in the revision and final approval of the manuscript.

Funding This work is supported by The National Natural Science Foundation of China (grant number 81774384), Fujian Key Laboratory of Rehabilitation Technology of China and Fujian provincial rehabilitation industrial institution of China (grant number 2015Y2001-55).

Competing interests None declared.

Patient consent for publication Obtained.

Ethics approval The study was approved by the Fujian University of Traditional Chinese Medicine Institutional Review Board (reference number: \#2018KY-006-1). 
Provenance and peer review Not commissioned; externally peer reviewed.

Data availability statement Data are available on reasonable request.

Open access This is an open access article distributed in accordance with the Creative Commons Attribution Non Commercial (CC BY-NC 4.0) license, which permits others to distribute, remix, adapt, build upon this work non-commercially, and license their derivative works on different terms, provided the original work is properly cited, appropriate credit is given, any changes made indicated, and the use is non-commercial. See: http://creativecommons.org/licenses/by-nc/4.0/.

\section{ORCID iDs}

Meijin Hou http://orcid.org/0000-0003-4830-5615

Shengxing Fu http://orcid.org/0000-0001-8983-6653

\section{REFERENCES}

1 Zeng S-ying, Gong Y, Zhang Y-ping, et al. Changes in the prevalence of rheumatic diseases in Shantou, China, in the past three decades: a COPCORD study. PLoS One 2015;10:e138492.

2 Barbour KE, Stevens JA, Helmick CG, et al. Falls and fall injuries among adults with arthritis - United States, 2012. MMWR Morb Mortal Wkly Rep 2014;63.

3 Hashish R, Toney-Bolger ME, Sharpe SS, et al. Texting during stair negotiation and implications for fall risk. Gait Posture 2017;58:409-14.

4 lijima H, Shimoura K, Aoyama T, et al. Biomechanical characteristics of stair ambulation in patients with knee oa: a systematic review with meta-analysis toward a better definition of clinical hallmarks. Gait Posture 2018;62:191-201.

5 Hinman RS, Bennell KL, Metcalf BR, et al. Delayed onset of quadriceps activity and altered knee joint kinematics during stair stepping in individuals with knee osteoarthritis. Arch Phys Med Rehabil 2002;83:1080-6.

6 Mat S, Ng CT, Tan MP. Influence of hip and knee osteoarthritis on dynamic postural control parameters among older fallers. J Rehabil Med 2017;49:258-63.

7 Manlapaz DG, Sole G, Jayakaran P, et al. Risk factors for falls in adults with knee osteoarthritis: a systematic review. $P m R$ 2019;11:745-57.

8 Sherrington C, Fairhall NJ, Wallbank GK, et al. Exercise for preventing falls in older people living in the community. Cochrane Database Syst Rev 2019;1.

9 Ghandali E, Moghadam ST, Hadian MR, et al. The effect of tai chi exercises on postural stability and control in older patients with knee osteoarthritis. J Bodyw Mov Ther 2017;21:594-8.

$10 \mathrm{Xu} \mathrm{D}$, Hong Y, Li J, et al. Effect of tai chi exercise on proprioception of ankle and knee joints in old people. Br J Sports Med 2004;38:50-4.

11 Su Z, Zhao J, Wang N, et al. Effects of weighted tai chi on leg strength of older adults. J Am Geriatr Soc 2015;63:2208-10.

12 Hochberg MC, Altman RD, April KT, et al. American College of rheumatology 2012 recommendations for the use of nonpharmacologic and pharmacologic therapies in osteoarthritis of the hand, hip, and knee. Arthritis Care Res 2012;64:465-74.

13 Lomas-Vega R, Obrero-Gaitán E, Molina-Ortega FJ, et al. Tai chi for risk of falls. A meta-analysis. J Am Geriatr Soc 2017;65:2037-43.

14 Andersson G, Hagman J, Talianzadeh R, et al. Effect of cognitive load on postural control. Brain Res Bull 2002;58:135-9.

15 Ghai S, Ghai I, Effenberg AO. Effects of dual tasks and Dual-task training on postural stability: a systematic review and meta-analysis. Clin Interv Aging 2017;12:557-77.

16 Wayne PM, Hausdorff JM, Lough M, et al. Tai chi training may reduce dual task gait variability, a potential mediator of fall risk, in healthy older adults: cross-sectional and randomized trial studies. Front Hum Neurosci 2015;9:332.

17 Vallabhajosula S, Tan CW, Mukherjee M, et al. Biomechanical analyses of stair-climbing while dual-tasking. J Biomech 2015;48:921-9.

18 Kelly VE, Janke AA, Shumway-Cook A. Effects of instructed focus and task difficulty on concurrent walking and cognitive task performance in healthy young adults. Exp Brain Res 2010;207:65-73.
19 Hamacher D, Rudolf M, Lohmann C, et al. Pain severity reduction in subjects with knee osteoarthritis decreases motor-cognitive Dualtask costs. Clin Biomech 2016;39:62-4.

20 Levinger $\mathrm{P}$, Nagano H, Downie C, et al. Biomechanical balance response during induced falls under dual task conditions in people with knee osteoarthritis. Gait Posture 2016;48:106-12.

21 Chan A-W, Tetzlaff JM, Altman DG, et al. Spirit 2013 statement: defining standard protocol items for clinical trials. Ann Intern Med 2013;158:200-7.

22 Chinese Rheumatology Association. Guideline for diagnosis and treatment of osteoarthritis. Chinese J Rheu 2010;14:416-9.

23 Bennell KL, Egerton T, Wrigley TV, et al. Comparison of neuromuscular and quadriceps strengthening exercise in the treatment of varus malaligned knees with medial knee osteoarthritis: a randomised controlled trial protocol. BMC Musculoskelet Disord 2011;12:276.

24 Wang C, Iversen MD, McAlindon T, et al. Assessing the comparative effectiveness of tai chi versus physical therapy for knee osteoarthritis: design and rationale for a randomized trial. BMC Complement Altern Med 2014;14:333.

25 Zhen LI. Research on Using the Priority of Learning and Cooperation Teaching Model of Eight-style Taijiquan"lianyi" to Improve the Effect of Taijiquan Teaching. Journal of Yichun College 2009.

26 Lesinski M, Hortobágyi T, Muehlbauer T, et al. Effects of balance training on balance performance in healthy older adults: a systematic review and meta-analysis. Sports Med 2015;45:1721-38

27 Levinger P, Dunn J, Bifera N, et al. High-Speed resistance training and balance training for people with knee osteoarthritis to reduce falls risk: study protocol for a pilot randomized controlled trial. Trials 2017;18:384.

28 Plummer P, Zukowski LA, Giuliani C, et al. Effects of physical exercise interventions on Gait-Related Dual-task interference in older adults: a systematic review and meta-analysis. Gerontology 2015;62:94-117.

29 Nascimbeni A, Minchillo M, Salatino A, et al. Gait attentional load at different walking speeds. Gait Posture 2015;41:304-6.

30 Patel P, Lamar M, Bhatt T. Effect of type of cognitive task and walking speed on cognitive-motor interference during Dual-task walking. Neuroscience 2014;260:140-8.

31 van Vugt Y, Stinear J, Claire Davies T, et al. Postural stability during gait for adults with hereditary spastic paraparesis. J Biomech 2019;88:12-17.

32 You H, Zhang H, Liu J, et al. Effect of balance training with Pro-kin system on balance in patients with white matter lesions. Medicine 2017;96:e9057

$33 \mathrm{JP}$ H, WC Y, RY L, et al. A preliminary study of test reliabilty in quantitative proprioception measurement. Chinese Journal of Physical Medicine and Rehabilitation 2012;34:34-7.

34 Schulz BW, Lloyd JD, Lee WE. The effects of everyday concurrent tasks on overground minimum toe clearance and gait parameters. Gait Posture 2010;32:18-22.

35 Begg R, Best R, Dell'Oro L, et al. Minimum foot clearance during walking: strategies for the minimisation of trip-related falls. Gait Posture 2007;25:191-8.

36 Bellamy N, Buchanan WW, Goldsmith $\mathrm{CH}$, et al. Validation study of WOMAC: a health status instrument for measuring clinically important patient relevant outcomes to antirheumatic drug therapy in patients with osteoarthritis of the hip or knee. J Rheumatol 1988;15:1833-40.

37 McConnell S, Kolopack P, Davis AM. The Western Ontario and McMaster universities osteoarthritis index (WOMAC): a review of its utility and measurement properties. Arthritis Rheum 2001;45:453-61.

38 Ettinger WH, Burns R, Messier SP, et al. A randomized trial comparing aerobic exercise and resistance exercise with a health education program in older adults with knee osteoarthritis. The fitness arthritis and seniors trial (fast). JAMA 1997;277:25-31.

39 Liao C-D, Lin L-F, Huang Y-C, et al. Functional outcomes of outpatient balance training following total knee replacement in patients with knee osteoarthritis: a randomized controlled trial. Clin Rehabil 2015;29:855-67. 